\title{
Administration of granulocyte colony-stimulating factor with radiotherapy promotes tumor growth by stimulating vascularization in tumor-bearing mice
}

\author{
JOONG SUN KIM ${ }^{1,5^{*}}$, YEONGHOON SON ${ }^{1 *}$, MIN JI BAE ${ }^{1}$, MINYOUNG LEE ${ }^{3}$, CHANG GEUN LEE ${ }^{1}$, \\ WOL SOON JO ${ }^{1}$, SUNG DAE KIM ${ }^{1}$ and KWANGMO YANG ${ }^{1,2,4}$
}

${ }^{1}$ Research Center and ${ }^{2}$ Department of Radiation Oncology, Dongnam Institute of Radiological and Medical Sciences
(DIRAMS), Busan 619-953; ${ }^{3}$ College of Pharmacy, Kyungpook National University, Daegu 702-701;
${ }^{4}$ Department of Radiation Oncology, Korea Institute of Radiological and Medical Sciences (KIRMAS), Seoul 139-706;
${ }^{5}$ College of Veterinary Medicine, Chonnam National University, Gwangju 500-757, Republic of Korea

Received February 4, 2015; Accepted April 14, 2015

DOI: 10.3892/or.2015.3977

\begin{abstract}
Although granulocyte-colony stimulating factor (G-CSF) is commonly used to support recovery from radiation-induced side-effects, the precise effects of G-CSF on colon cancer under radiotherapy remain poorly understood. In the present study, to investigate the effects of tumor growth following radiotherapy and G-CSF administration in a murine xenograft model of colon cancer, female BALB/c mice were injected with cells of a colon carcinoma cell line (CT26) with irradiation and G-CSF, alone or in combination. Mice received 2 Gy of focal radiation daily for 5 days and intraperitoneal injection of G-CSF (100 $\mu \mathrm{g} / \mathrm{kg} /$ day) after irradiation for 7 days. Changes in the levels of myeloperoxidase (MPO), vascular endothelial growth factor (VEGF), matrix metalloproteinase type 9 (MMP-9) and CD31 were assessed in the mouse cancer induced by injection of colon cancer cells. We observed that G-CSF increased the number of circulating neutrophils, but facilitated tumor growth. However, G-CSF treatment did not affect radiation-induced cytotoxicity and cell viability in CT26 cells in vitro. Increased levels of myeloperoxidase, a neutrophil marker and those of vascular endothelial growth factor were observed in tumors with G-CSF supplementation. In addition, we found that increased levels of CD31 and matrix metalloproteinase-9 were correlated with the enhanced tumor growth after G-CSF treatment. Therefore, these data suggest that G-CSF may contribute to tumor growth and decrease the antitumor effect of radiotherapy, possibly by promoting vascularization in cancer lesions.
\end{abstract}

Correspondence to: Dr Joong Sun Kim, Research Center, Dongnam Institute of Radiological and Medical Sciences (DIRAMS), Gijang-gun, Busan 619-953, Republic of Korea

E-mail: jskim@dirams.re.kr

*Contributed equally

Key words: G-CSF, irradiation, colon cancer, vascularization

\section{Introduction}

Radiotherapy is widely employed in the clinic to treat a variety of cancers. However, since ionizing radiation also affects normal cells, side-effects are commonly observed in patients receiving radiotherapy. An important adverse effect induced by radiotherapy and chemotherapy is myelosuppression, with a significant decline in the number of circulating neutrophils $(1,2)$. However, no effective treatment has been developed to mitigate the radiation-induced injury.

Granulocyte colony stimulating factor (G-CSF), an agent clinically approved for neutropenia treatment, is often used to increase the number of neutrophils after radiation and chemotherapy $(3,4)$. Furthermore, the ability of G-CSF to reverse myelosuppression following radiation exposure has been demonstrated in animal models (5). In addition to the hematopoietic functions, G-CSF has been suggested to offer protective effects in other tissues, including the liver (6), brain (7) and intestine (8). Moreover, a recent in vivo study reported that G-CSF treatment administered before or after irradiation attenuated focal irradiation-induced intestinal mucosal damage $(9,10)$. G-CSF also ameliorated radiation-induced apoptosis in intestinal epithelial cells in vitro (10). Although G-CSF may have a radio-protective effect against gastrointestinal damage after irradiation exposure, its exact influence on cancer cells after irradiation remain poorly understood.

The induction of neutrophilia by G-CSF may have antitumor effects in cancer patients (11). Additionally, in the experimental animals, antitumor effects by G-CSF have been observed in a murine xenograft model of human medulloblastoma (12). In contrast, G-CSF has been shown to stimulate cancer angiogenesis and promote tumor growth (13). G-CSF treatment with chemotherapy has also been suggested to facilitate revascularization and enhance tumor growth (14). Thus, the effects of G-CSF on tumor growth, especially under irradiation therapy, are controversial and require further investigation. Nevertheless, the precise involvement of G-CSF treatment in colon cancer following radiotherapy remains unknown.

Therefore, the present study evaluated tumor growth following radiotherapy and G-CSF administration in a murine 
xenograft model of colon cancer. Changes in the expression levels of myeloperoxidase (MPO), vascular endothelial growth factor (VEGF), matrix metalloproteinase-9 (MMP-9) and CD31 were also assessed in the mouse cancer induced by injection of colon cancer cells.

\section{Materials and methods}

Cell culture. CT26 BALB/c mouse colon cancer cells (Korean Cell Line Bank, Seoul, Korea) were cultured in the Roswell Park Memorial Institute 1640 medium (Nissui Pharmaceutical Co., Tokyo, Japan) containing $10 \%$ heat-inactivated fetal bovine serum, 100 units $/ \mathrm{ml}$ penicillin, $0.1 \mathrm{mg} / \mathrm{ml}$ streptomycin and $0.5 \mathrm{mM}$ glutamine (Invitrogen, Grand Island, NY, USA). Cells were incubated at $37^{\circ} \mathrm{C}$ in a humidified atmosphere with $5 \% \mathrm{CO}_{2}$.

Animal experiments. Female BALB/c mice (6-week-old) were purchased from the Central Lab. Animal Inc. (Seoul, Korea) and used after one week of quarantine and acclimatization. All animals were maintained in a room at $23 \pm 2{ }^{\circ} \mathrm{C}$ with a relative humidity of $50 \pm 5 \%$, artificial lighting from 08:00 to 20:00 daily and 13-18 air changes/hour. They had ad libitum access to standard laboratory diet and water. After acclimatization, mice were randomly divided into four groups ( $\mathrm{n}=6 \mathrm{mice} /$ group): control, treatment with radiation, treatment with G-CSF, and treatment with radiation and G-CSF. CT26 cells $\left(1 \times 10^{6}\right.$ cells/animal in $100 \mu \mathrm{l}$ PBS) were injected subcutaneously in the right flank of BALB/c mice. Tumor volume was measured at 7,14,17, and 21 days after implantation using calipers. At the end of the study, the tumor of each animal was quickly frozen and immediately stored for protein extraction ( $n=4$ /group) or fixed in buffered formalin for immunohistochemistry ( $\mathrm{n}=2$ /group).

All experimental procedures were conducted according to the National Institutes of Health Guidelines for the Care and Use of Laboratory Animals and following a protocol approved by the Institutional Animal Care and Use Committee of the Dongnam Institute of Radiological and Medical Sciences. All animals were cared for in accordance with the National Animal Welfare Law of Korea.

Irradiation and G-CSF treatment. A schematic schedule for radiation and G-CSF treatments is shown in Fig. 1A. When tumors reached a mean volume of $\sim 100 \mathrm{~mm}^{3}$ (day 7), mice received sham or focal radiation. Anesthetized mice were positioned on a tray with the intestine and cancer lesion in the radiation field. Partial body irradiation was administered at a daily dose of 2 Gy for 5 days using 6-MV high-energy photon rays (Elekta, Stockholm, Sweden) at a dose rate of $3.8 \mathrm{~Gy} / \mathrm{min}$. Sham-irradiated mice underwent similar procedures without radiation.

Recombinant human G-CSF (Neutrogin, Choongwae Pharm. Co., Seoul, Korea) was dissolved in $0.9 \%$ saline and injected intraperitoneally at $100 \mu \mathrm{g} / \mathrm{kg}$ for 7 days from the day of radiation treatment. Sham-treated mice received the same volume of saline.

Blood collection. Blood samples were collected from the vena cava into tubes containing ethylenediaminetetraacetic acid.
Peripheral white blood cells (WBC) and neutrophils were monitored by automatic counting using a Hemavet (Drew Scientific Inc., UK).

Immunohistochemistry. Tissue samples were processed in paraffin, cut into $4-\mu \mathrm{m}$ thick coronal sections and deparaffinized. The sections were incubated in normal goat serum (Vector ABC Elite kit; Vector Laboratories Inc., Burlingame, CA, USA) for $60 \mathrm{~min}$ to prevent non-specific binding, followed by incubation with rabbit monoclonal anti-MPO (1:200; Cell Signaling Technology, Beverly, MA, USA), rabbit monoclonal anti-CD31 (1:1,000; Cell Signaling Technology), rabbit monoclonal anti-MMP-9 (1:200; Acris Antibodies GmbH, Herford, Germany) for $2 \mathrm{~h}$ at room temperature (RT). Then, the sections were reacted with biotinylated goat anti-rabbit IgG (Vector ABC Elite kit) for $60 \mathrm{~min}$ at RT. Immunoreactivity was performed for $60 \mathrm{~min}$ at RT using the avidin-biotin peroxidase complex (Vector ABC Elite kit) prepared according to the manufacturer's instructions and the peroxidase reaction was developed using a diaminobenzidine substrate kit (SK-4100; Vector). For controls, incubation with the primary antibodies was omitted. All sections were counterstained with Harris' hematoxylin prior to being mounted.

Western blot analysis. Harvested tumor tissues were homogenized in lysis buffer and the protein concentration was determined using a Bio-Rad protein assay kit (Bio-Rad Laboratories, Hercules, CA, USA) with bovine serum albumin set as the standard. A sodium dodecyl sulfate sample buffer (4X) was added to each homogenized sample and the samples were heated at $100^{\circ} \mathrm{C}$ for $10 \mathrm{~min}$. The samples were then separated by electrophoresis, transferred to a polyvinylidene difluoride membrane, and blocked with 5\% skim milk in phosphate-buffered saline containing 0.1\% Tween 20 (PBS-T) for $30 \mathrm{~min}$ at RT. The membranes were incubated with rabbit monoclonal anti-MPO (1:200; Cell Signaling Technology), anti-VEGF (1:1,000; Cell Signaling Technology), rabbit monoclonal anti-CD31 (1:1,000; Cell Signaling Technology) and rabbit monoclonal anti-MMP-9 (1:200; Acris Antibodies $\mathrm{GmbH}$ ) in PBS-T overnight at $4^{\circ} \mathrm{C}$. After extensive washing and incubation with horseradish peroxidase-conjugated goat anti-rabbit IgG (1:20,000; Pierce, Rockford, IL, USA), the signals were visualized using chemiluminescence method (SuperSignal West Pico; Pierce). For normalization of expression, the membranes were incubated with a mouse monoclonal antibody for $\beta$-actin (1:20,000; Sigma-Aldrich, St. Louis, MO, USA) and processed as mentioned above. Several exposure times were used to obtain signals in a linear range. Band intensities were quantified using Scion Image Beta 4.0.2 for Windows XP software (Scion Corp., Frederick, MD, USA). Values shown represent the mean \pm standard error $(\mathrm{SE})$.

Cytotoxicity and cell viability evaluation. Cytotoxicity was measured using a lactate dehydrogenase (LDH) cytotoxicity assay kit (BioVision, Mountain View, CA, USA) according to the manufacturer's recommendations. The assay was assessed quantitatively via the measurement of LDH, which was released from damaged or destroyed cells in the extracellular fluid. The LDH activity was quantified by the colorimetric reduction of a tetrazolium salt (yellow) to formazan (red). 

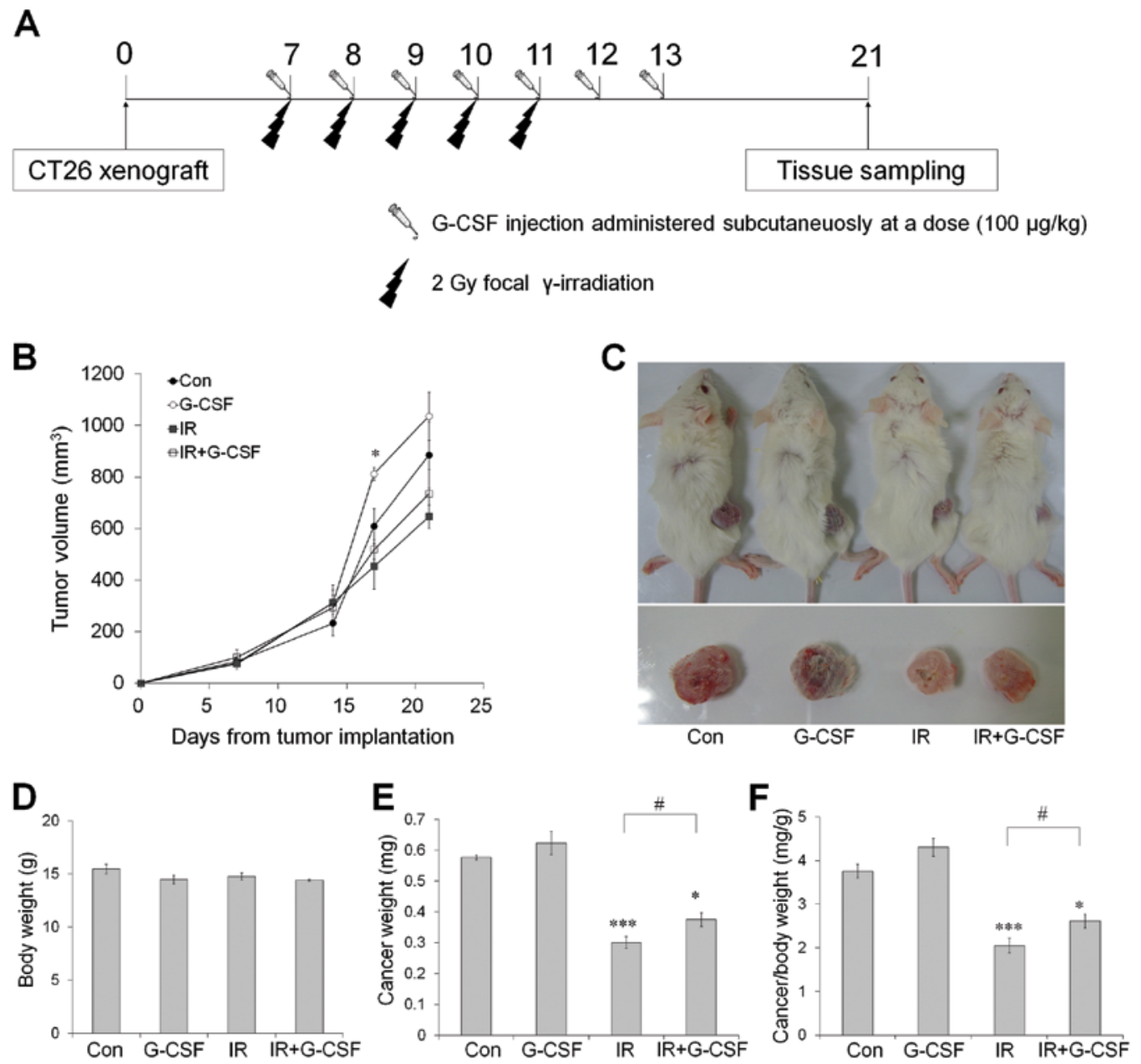

Figure 1. CT26 tumor growth in BALB/c mice treated with radiation and G-CSF, alone or in combination. (A) Schematic diagram of the experimental procedure, (B) Tumor volumes measured by calipers at 7, 14, 17 and 21 days after implantation, (C) Representative photographs of tumor-bearing mice treated with radiation and G-CSF, alone or in combination, 21 days after tumor implantation. Before tissue sampling, the body weight (D), tumor weight (E) and relative tumor weight $(\mathrm{F})$ were calculated. Data are reported as means $\pm \mathrm{SE}$ (n=6/group). ${ }^{*} \mathrm{p}<0.05,{ }^{* *} \mathrm{p}<0.01,{ }^{* * *} \mathrm{p}<0.001$ vs. sham controls; ${ }^{*} \mathrm{p}<0.05$ vs. sham-treated irradiated groups. G-CSF, granulocyte-colony stimulating factor.

After a 30-min incubation at RT, absorbance at $450 \mathrm{~nm}$ was measured to determine LDH activity. Data shown are the mean \pm standard error (SE) of 4 cultures/condition. Blank LDH levels were subtracted from the LDH values for each sample and the results were normalized to $100 \%$.

Cell viability was evaluated using a 3-(4,5-dimethylthiazol2-yl)-2,5-diphenyl tetrazolium bromide (MTT) assay (Sigma Chemicals Co.). The assay was based on the reduction of MTT by living cells to yield a soluble formazan product that could be detected colorimetrically. MTT was added to cultured cells at $0.6 \mathrm{mg} / \mathrm{ml}$ culture medium without phenol red. Cell plates were incubated at $37^{\circ} \mathrm{C}$ for $4 \mathrm{~h}$ before the MTT solution was removed and cells were dissolved in a solubilization solution (dimethyl sulfoxide and ethanol, 1:1 ratio). The absorbance of solubilized formazan product was quantified by a microplate reader at $560 \mathrm{~nm}$. Data shown are the mean $\pm \mathrm{SE}$ of 4 replicates/condition. Blank MTT levels were subtracted from the MTT values for each sample and the results were normalized to $100 \%$.

Statistical analysis. The data are reported as the mean $\pm \mathrm{SE}$ and were analyzed using one-way analysis of variance followed by the Student-Newman-Keuls post-hoc test for multiple comparisons. A p-value of $<0.05$ was considered significant.

\section{Results}

$G$-CSF induces tumor growth in BALB/c mice bearing CT26 xenografts. The potential clinical implications of G-CSF combined with radiation were assessed in vivo using a CT26 xenograft model. Tumor-bearing mice were sham-irradiated or received a total dose of $10 \mathrm{~Gy}$, with or without G-CSF at a dosage of $100 \mu \mathrm{g} / \mathrm{kg} / \mathrm{day}$. All treatments were well tolerated, with no signs of toxicity observed or gross pathologic abnormalities noted on necropsy. Tumors exposed to fractionated radiation were significantly smaller than those sham-irradiated ( $<<0.01$ at 21 days after tumor implantation) (Fig. 1B). However, tumors treated with G-CSF alone significantly increased in size at 17 days after implantation (Fig. 1B). Tumors exposed to radiation with G-CSF had significantly increased absolute and relative tumor weight on day 21 after implantation compared with those receiving radiation only (Fig. $1 \mathrm{E}$ and $\mathrm{F}$ ). Thus, these data indicate that G-CSF may support tumor growth and interfere with antitumor effect of radiotherapy. 
A

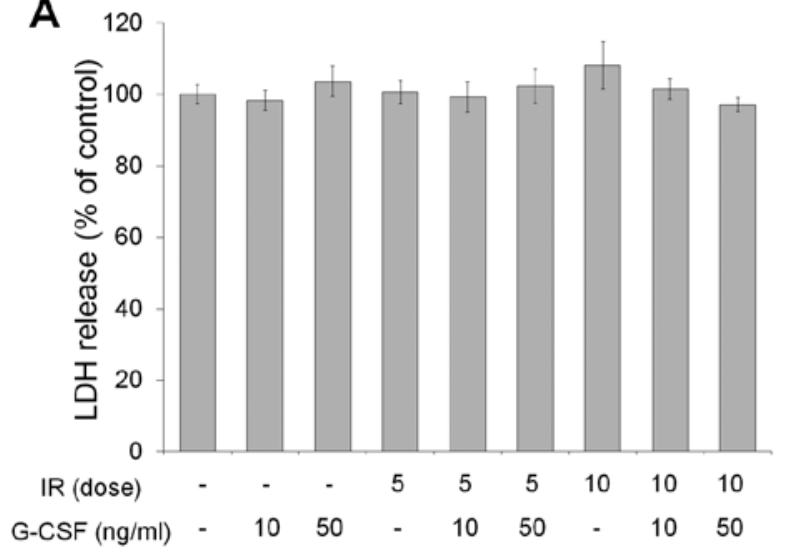

C

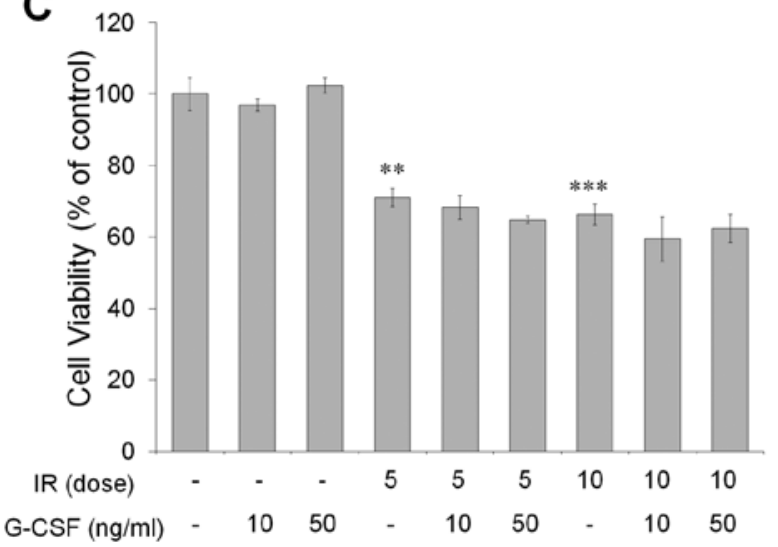

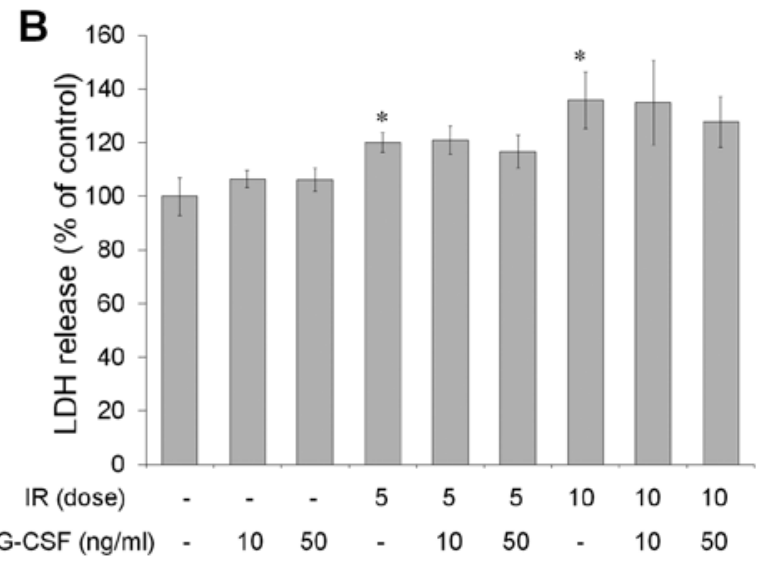

D

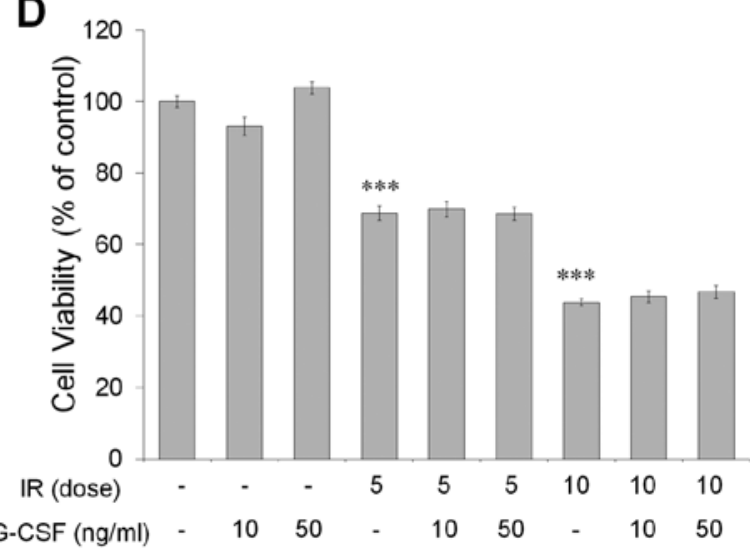

Figure 2. Evaluation of cytotoxicity and cell viability in CT26 cells after treatment with radiation and G-CSF, alone or in combination. Cytotoxicity was detected by lactate dehydrogenase release at 48 and $72 \mathrm{~h}$ (A and B) after treatment with radiation or G-CSF. Cell viability was detected using the 3-(4,5-dimethylthiazol-2-yl)-2,5-diphenyl tetrazolium bromide assay at 48 and $72 \mathrm{~h}(\mathrm{C}$ and D) after treatment. Data are reported as means $\pm \mathrm{SE}$ ( $\mathrm{n}=4 / \mathrm{group}) .{ }^{*} \mathrm{p}<0.05$, ${ }^{* * *} \mathrm{p}<0.01,{ }^{* * * *} \mathrm{p}<0.001$ vs. sham controls. G-CSF, granulocyte-colony stimulating factor.
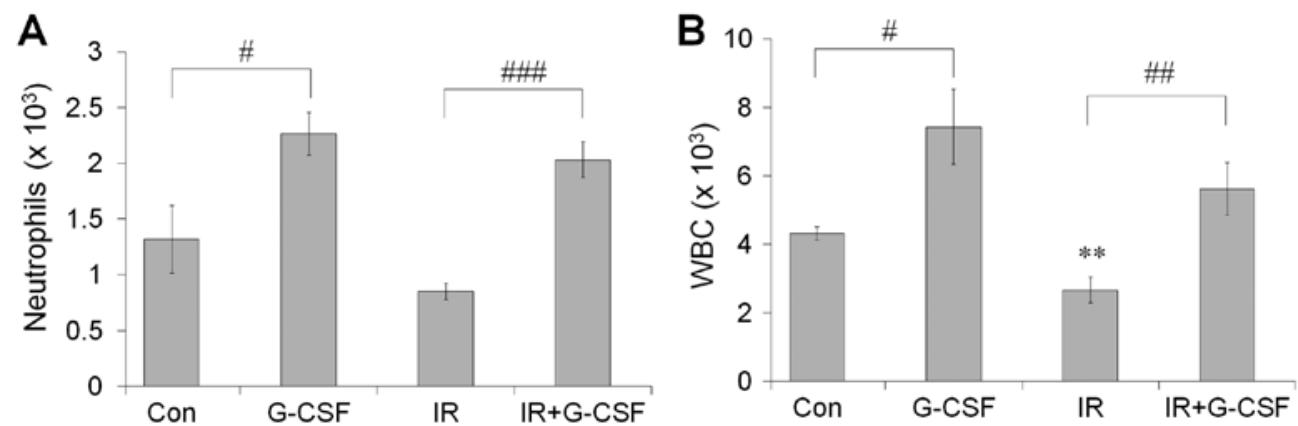

Figure 3. Changes in the number of circulating neutrophils (A) and white blood cells (B) after radiation, granulocyte-colony stimulating factor or both. Data are reported as means $\pm \mathrm{SE}$ ( $\mathrm{n}=6 /$ group). ${ }^{* *} \mathrm{p}<0.01 \mathrm{vs}$. sham controls; ${ }^{\#} \mathrm{p}<0.05,{ }^{\# \#} \mathrm{p}<0.01,{ }^{\# \# \#} \mathrm{p}<0.001$ vs. sham-treated irradiated groups.

$G$-CSF does not affect radiation-induced cytotoxicity and cell viability in vitro. To examine the effect of G-CSF on radiation-induced cytotoxicity and cell viability, CT-26 cells were irradiated with 5 or $10 \mathrm{~Gy}$ and treated with or without $10-50 \mathrm{ng} / \mathrm{ml}$ of G-CSF. At $48 \mathrm{~h}$ after irradiation, cytotoxicity assessed by the LDH assay showed no changes by radiation or G-CSF treatment (Fig. 2A). However, at $72 \mathrm{~h}$, significantly increased cytotoxicity was observed in cells receiving radiation, but G-CSF did not exert such an effect compared to sham-treated controls (Fig. 2B). Although cell viability, evalu- ated by the MTT assay, decreased significantly with radiation, G-CSF treatment did not alter cell viability at 48 and $72 \mathrm{~h}$ after irradiation (Fig. 2C and D). Thus, irradiation-induced cytotoxicity and cell viability were not altered by G-CSF treatment.

$G$-CSF increased the number of peripheral WBCs and neutrophils. The number of circulating WBCs and neutrophils was assessed 21 days after radiotherapy to evaluate the effect of radiotherapy on blood counts (Fig. 3). We found, as expected, that WBCs and neutrophils were decreased after irradiation. 


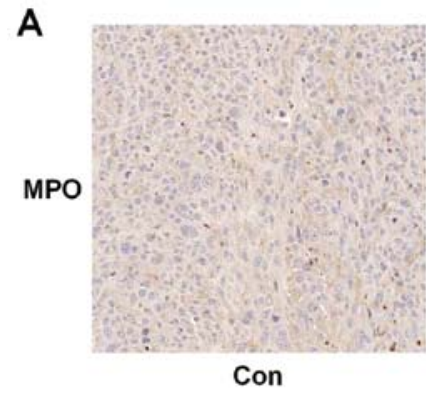

B

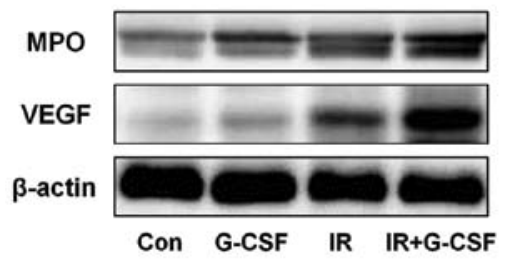

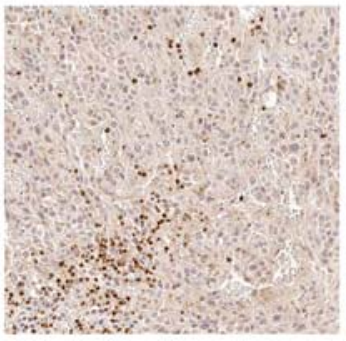

G-CSF

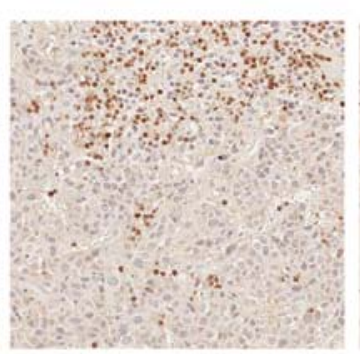

IR

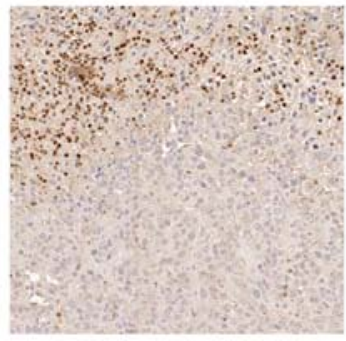

IR+G-CSF
C

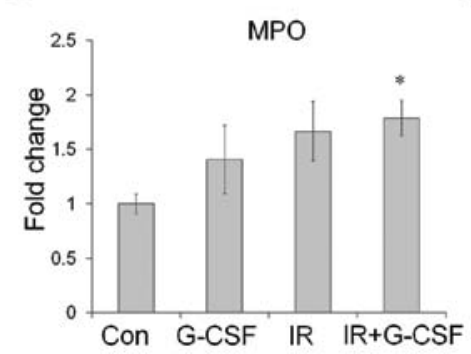

D

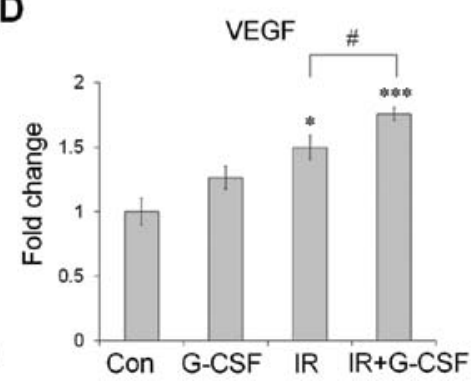

Figure 4. Relative expression of MPO and VEGF in tumors after radiation, granulocyte-colony stimulating factor, or both. (A) Representative photographs of MPO expression in tumors 21 days after implantation. (B) Representative immunoblots for MPO and VEGF. The relative protein levels of MPO (C) and VEGF (D) were determined by densitometry and normalized to $\beta$-actin signals. Data are reported as means $\pm \mathrm{SE}$ ( $\mathrm{n}=4 / \mathrm{group}$ ). ${ }^{*} \mathrm{p}<0.05$, ${ }^{* * *} \mathrm{p}<0.001$ vs. sham controls; ${ }^{\text {p }}<0.05$ vs. sham-treated irradiated groups. MPO, myeloperoxidase; VEGF, vascular endothelial growth factor.

However, the number of WBCs and neutrophils increased significantly following G-CSF treatment at 21 days after irradiation. Thus, G-CSF treatment induced the upregulation of circulation WBCs and neutrophils in tumor-bearing mice.

The expression levels of MPO and VEGF are upregulated in tumors treated with radiation and G-CSF. Next, we assessed MPO, a marker for neutrophils, to determine whether G-CSF induced the recruitment of neutrophils into the tumor after irradiation (Fig. 4). MPO protein levels were shown to increase after radiation by both immunohistochemistry and western blot analysis and G-CSF supplementation further promoted such an increase (Fig. 4A-C). VEGF protein levels, determined by western blot analysis, also increased after fractionated irradiation, and change in the patterns of VEGF were similar to those of MPO (Fig. 4D). These results indicate that radiation-induced neutrohphil infiltration accompanied by VEGF is aggravated by G-CSF treatment.

Irradiation-induced vascular damage is mitigated by G-CSF administration. To investigate the possible mechanisms involved in tumor growth after G-CSF treatment, CD31 and MMP-9 levels were assessed (Fig. 5). CD31 expression levels were reduced significantly after irradiation as determined by western blot analysis and the number of CD31-positive cells also decreased in tumor tissue as determined by immunohistochemistry (Fig. 5A and C). After G-CSF supplementation, CD31 levels increased significantly compared to vehicletreated controls with or without irradiation. MMP-9 levels did not change after irradiation monotherapy. However, western blot analysis data showed that G-CSF treatment significantly increased MMP-9 levels (Fig. 5D) and comparable result was also observed by immunohistochemistry in the tumor tissue (Fig. 5A), although the differences between radiation with G-CSF and radiation only were not significant. These data suggest that G-CSF supplementation may decrease radiationinduced vascular damages and increase MMP-9 expression levels in tumors.

\section{Discussion}

The present study demonstrates that G-CSF may attenuate the antitumor effect of radiotherapy. Although G-CSF may induce neutrophil upregulation via bone marrow stimulation, it could also promote tumor vascularization, possibly via production of pro-angiogenic factors.

Radiotherapy is a commonly used method to treat tumors in cancer patients. Because G-CSF is known to increase neutrophil production by mobilizing hematopoietic stem cells from the bone marrow, it is often used clinically to treat neutropenia induced by chemotherapy or radiotherapy $(15,16)$. In experimental animals, a previous study by us, and other showed the protective effects of G-CSF against radiation-induced injury of the normal intestine both in vivo and in vitro $(10,17)$. Additionally, G-CSF treatment after irradiation has been shown to enhance survival and minimize the effects of radiation on gastrointestinal injury in mice (9). Furthermore, there is evidence that G-CSF may have a therapeutic effect induced by brain irradiation by inhibiting the detrimental effects on hippocampal neurogenesis (7).

The effects of tumor by irradiation can be different between high single dose and small fractionated dose. Although high single dose of irradiation is more effective in tumor treatment (18), this schedule is not equivalent to the clinical fractionated radiation protocol ( $\sim 2 \mathrm{~Gy} /$ fraction). It was reported that the effects of G-CSF was dose-dependent, however, the number of neutrophil counts in irradiated mice treated with 300 and $600 \mu \mathrm{g} / \mathrm{kg}$ were higher than that of neutrophil counts 

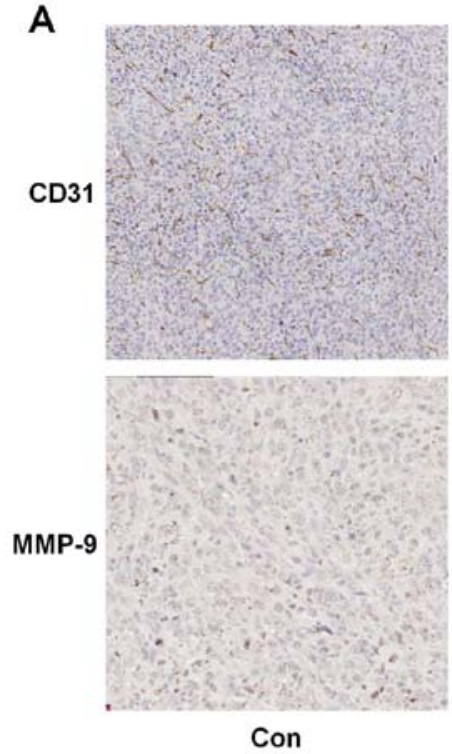
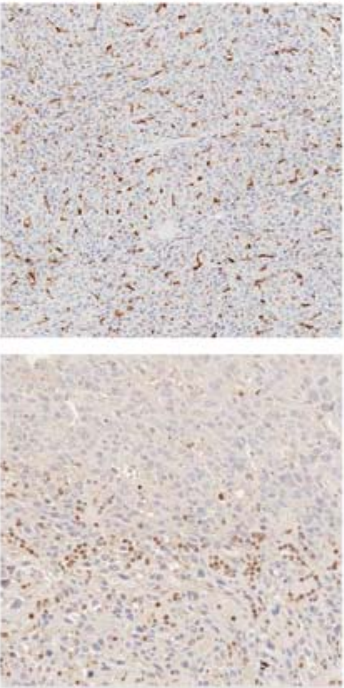

G-CSF
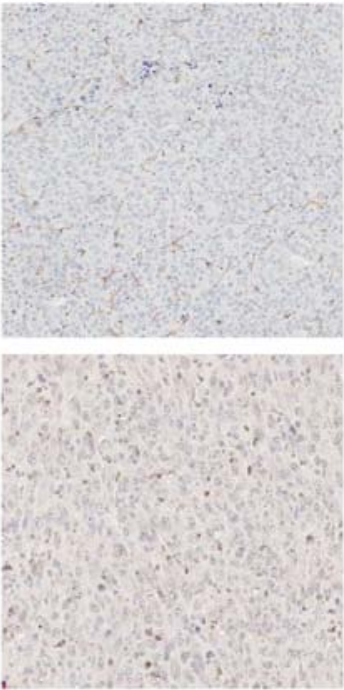

IR
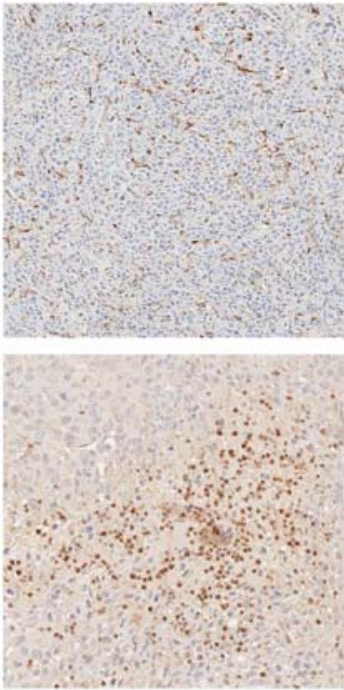

IR+G-CSF
B

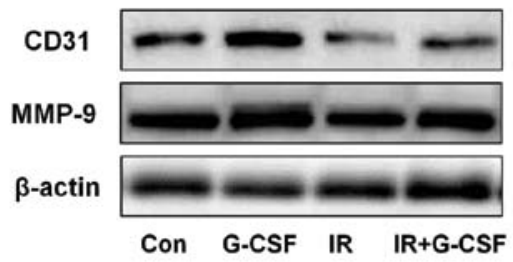

C

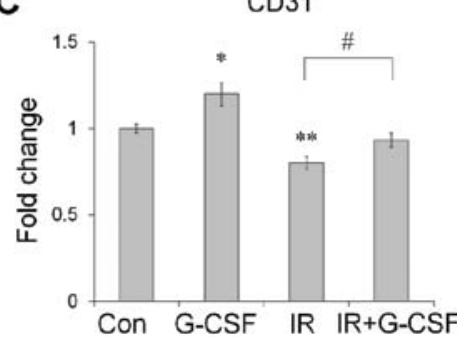

D

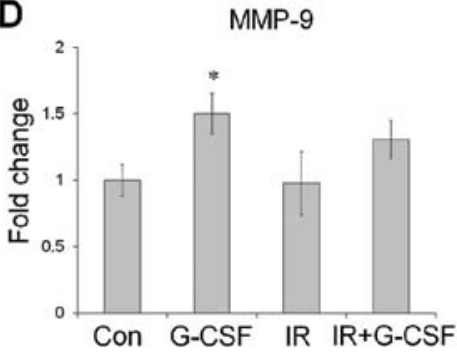

Figure 5. Relative expression of CD31 and MMP-9 in tumors after radiation, granulocyte-colony stimulating factor or both. (A) Representative photographs of CD31 and MMP-9 expression in tumors 21 days after implantation. (B) Representative immunoblots for CD31 and MMP-9. The relative protein levels of CD31 (C) and MMP-9 (D) were determined by densitometry and normalized to $\beta$-actin signals. Data are reported as means \pm SE ( $\mathrm{n}=4 / \mathrm{group}){ }^{*} \mathrm{p}<0.05,{ }^{* *} \mathrm{p}<0.01$ vs. sham controls; ${ }^{\#} \mathrm{p}<0.05$ vs. sham-treated irradiated groups. MMP-9, matrix metalloproteinase-9.

in sham-irradiated control (19). Although many previous studies have used various G-CSF doses $(13,14,20)$, we used $\mathrm{G}-\mathrm{CSF}$ at $100 \mu \mathrm{g} / \mathrm{kg}$, which is consistent with our previous studies to determine the protective effect of G-CSF against radiation $(7,9)$. In a previous study, tumors were collected in the late tumor growth to examine the role of tumor vasculature by G-CSF (20). Further, tumors were excised when they reached a size of 10 to $12 \mathrm{~mm}$, which is relevant to $\sim 1,000 \mathrm{~mm}^{3}$ in size, to investigate vascular response with fractionated radiation protocol $(13,21)$. Therefore, in the present study, we have examined the side-effects and vascular changes in tumors in the late tumor growth phase following fractionated irradiation with G-CSF treatment.

Clinically, G-CSF, with or without chemotherapy, has demonstrated anticancer effects $(22,23)$. However, it is important to comment that the administration of G-CSF administration may also associate with cancer progression. Previously, it was reported that G-CSF may have potential to promote tumor growth in mice inoculated with colon cancer (13). Additionally, despite its known clinical benefits, G-CSF may attenuate the antitumor activity of chemotherapy (14). Therefore, the exact effects of G-CSF administration on cancer cells remain controversial. The mechanisms associated with tumor growth when exogenous G-CSF is administered with radiotherapy are also unknown.
Thus, this preclinical study aimed to determine whether G-CSF diminished the antitumor effects of radiation. We found that compared to radiation alone, combinational G-CSF administration attenuated the tumor suppression effects of radiotherapy, suggesting that G-CSF may contribute to tumor growth after irradiation.

The use of G-CSF supplementation in neutropenic cancer patients treated with radiation or chemotherapy have been suggested. Because it is well-known that G-CSF administration increases the production of neutrophils, it was suggested that cytotoxic mediators produced by neutrophils could kill the cancer cells $(24,25)$. In the present study, as expected, we confirmed that G-CSF increased the number of circulating neutrophils. Because it was previously reported that neutrophils can be identified by the cytoplasmic marker MPO coupled with cell morphology (26), we assessed MPO levels in xenograft tumors treated with radiation and G-CSF. Although irradiation alone led to increased expression levels of MPO, G-CSF treatment upregulated the number of neutrophils in tumors. G-CSF has been shown to promote angiogenesis by upregulating VEGF, which is released by neutrophils, but not by monocytes (27). In addition, it has been reported that VEGF signaling is required for vasculogenesis and angiogenesis (28), and tumor-produced VEGF not only promotes local angiogenesis but also mobilizes hematopoietic progenitors from 
the bone marrow $(29,30)$. In agreement with these previous findings, we showed that VEGF levels increased in tumors treated with both radiation and G-CSF compared to controls and those receiving radiation only. Moreover, the pattern of VEGF changes was similar to that of MPO. Our findings suggest that both radiation and G-CSF may associate with VEGF upregulation, possibly via increased neutrophil infiltration into tumors and radiotherapy combined with G-CSF may facilitate vascularization.

Tumor can expand its vasculature by angiogenesis, which results from nearby endothelial cell proliferation, or by vasculogenesis, which occurs via the recruitment of circulating endothelial and other cells from the bone marrow (31). Radiation has been reported to induce angiogenesis blockage by abrogating endothelial cells (32), thereby forcing the tumor to rely on vasculogenesis promoted by increased hypoxia (31). We demonstrated that CD31-positive endothelial cells in the tumor were significantly decreased following irradiation; G-CSF, however, reduced such radiation-induced vascular damage. MMP-9 is involved in extracellular matrix degradation and vascular remodeling (33) and may enhance local angiogenesis by releasing angiogenic factors such as VEGF (34). Additionally, subcutaneously implanted tumors could not grow in MMP-9 knockout mice, but tumor growth was restored by transplantation of wild-type bone marrow, implicating vasculogenesis involvement (35). We found that G-CSF, regardless of radiation, elevated MMP-9 levels in tumors. Thus, despite radiation-induced endothelial abrogation in tumor, G-CSF may promote tumor growth by enhancing angiogenesis and vasculogenesis. Further studies are needed to verify the specific mechanisms underlying tumor growth after G-CSF treatment and associated vasculogenesis following ionizing irradiation.

In conclusion, the present study demonstrates that CT26 tumor growth in mice may be facilitated by G-CSF administered with radiotherapy. Although G-CSF increased neutrophils levels, it was accompanied by VEGF upregulation. Additionally, levels of vascularization-related factors characterized by CD31 and MMP-9 in tumors were correlated with increased tumor growth by G-CSF treatment. Consequently, we suggested that G-CSF treatment may enhance tumor growth after radiation, possibly via increasing levels of vascularization in the tumor. More comprehensive studies are needed to improve the outcomes of cancer patients receiving radiotherapy.

\section{Acknowledgements}

The present study was supported by the Nuclear R\&D Program of the Ministry of Education, Science and Technology, Korea (Grant no., 50496-2013).

\section{References}

1. Mauch P, Constine L, Greenberger J, Knospe W, Sullivan J, Liesveld JL and Deeg HJ: Hematopoietic stem cell compartment: Acute and late effects of radiation therapy and chemotherapy. Int J Radiat Oncol Biol Phys 31: 1319-1339, 1995.

2. Friberg LE, Henningsson A, Maas H, Nguyen L and Karlsson MO: Model of chemotherapy-induced myelosuppression with parameter consistency across drugs. J Clin Oncol 20: 4713-4721, 2002.

3. Sung L and Dror Y: Clinical applications of granulocyte-colony stimulating factor. Front Biosci 12: 1988-2002, 2007.
4. MacVittie TJ and Farese AM: Cytokine-based treatment of radiation injury: Potential benefits after low-level radiation exposure. Mil Med 167 (Suppl): 68-70, 2002.

5. Uckun FM, Souza L, Waddick KG, Wick M and Song CW: In vivo radioprotective effects of recombinant human granulocyte colony-stimulating factor in lethally irradiated mice. Blood 75: 638-645, 1990.

6. Hou XW, Jiang Y, Wang LF, Xu HY, Lin HM, He XY, He JJ and Zhang S: Protective role of granulocyte colony-stimulating factor against adriamycin induced cardiac, renal and hepatic toxicities. Toxicol Lett 187: 40-44, 2009.

7. Kim JS, Yang M, Jang H, Oui H, Kim SH, Shin T, Jang WS Lee SS and Moon C: Granulocyte-colony stimulating factor ameliorates irradiation-induced suppression of hippocampal neurogenesis in adult mice. Neurosci Lett 486: 43-46, 2010.

8. Kudo T, Matsumoto T, Nakamichi I, Yada S, Esaki M, Jo Y, Ohji Y, Yao T and Iida M: Recombinant human granulocyte colony-stimulating factor reduces colonic epithelial cell apoptosis and ameliorates murine dextran sulfate sodium-induced colitis. Scand J Gastroenterol 43: 689-697, 2008.

9. Kim JS, Ryoo SB, Heo K, Kim JG, Son TG, Moon C and Yang K: Attenuating effects of granulocyte-colony stimulating factor (G-CSF) in radiation induced intestinal injury in mice. Food Chem Toxicol 50: 3174-3180, 2012

10. Kim JS, Yang M, Lee CG, Kim SD, Kim JK and Yang K: In vitro and in vivo protective effects of granulocyte colony-stimulating factor against radiation-induced intestinal injury. Arch Pharm Res 36: 1252-1261, 2013.

11. Lopez-Lazaro M: Granulocyte colony-stimulating factor (G-CSF): A novel anticancer therapy based on the 'universal dynamics of tumor growth'? Exp Oncol 28: 249-251, 2006.

12. Maeda H, Uozumi T, Kurisu K, Matsuoka T, Kawamoto K, Kiya K, Ogasawara H, Sugiyama K, Mikami T, Monden S, et al: Combined antitumor effects of TNF and G-CSF on a human medulloblastoma xenograft line. J Neurooncol 21: 203-213, 1994.

13. Natori T, Sata M, Washida M, Hirata Y, Nagai R and Makuuchi M: G-CSF stimulates angiogenesis and promotes tumor growth: Potential contribution of bone marrow-derived endothelial progenitor cells. Biochem Biophys Res Commun 297: 1058-1061, 2002.

14. Voloshin T, Gingis-Velitski S, Bril R, Benayoun L, Munster M, Milsom C, Man S, Kerbel RS and Shaked Y: G-CSF supplementation with chemotherapy can promote revascularization and subsequent tumor regrowth: Prevention by a CXCR4 antagonist. Blood 118: 3426-3435, 2011.

15. Roberts AW: G-CSF: A key regulator of neutrophil production, but that's not all! Growth Factors 23: 33-41, 2005.

16. Hérodin F and Drouet M: Cytokine-based treatment of accidentally irradiated victims and new approaches. Exp Hematol 33: 1071-1080, 2005.

17. Singh VK, Fatanmi OO, Singh PK and Whitnall MH: Role of radiation-induced granulocyte colony-stimulating factor in recovery from whole body gamma-irradiation. Cytokine 58: 406-414, 2012

18. Garcia-Barros M, Paris F, Cordon-Cardo C, Lyden D, Rafii S, Haimovitz-Friedman A, Fuks Z and Kolesnick R: Tumor response to radiotherapy regulated by endothelial cell apoptosis. Science 300: 1155-1159, 2003.

19. Romero-Weaver AL, Wan XS, Diffenderfer ES, Lin L and Kennedy AR: Kinetics of neutrophils in mice exposed to radiation and/or granulocyte colony-stimulating factor treatment. Radiat Res 180: 177-188, 2013.

20. Shojaei F, Wu X, Qu X, Kowanetz M, Yu L, Tan M, Meng YG and Ferrara N: G-CSF-initiated myeloid cell mobilization and angiogenesis mediate tumor refractoriness to anti-VEGF therapy in mouse models. Proc Natl Acad Sci USA 106: 6742-6747, 2009.

21. Chen FH, Fu SY, Yang YC, Wang CC, Chiang CS and Hong JH: Combination of vessel-targeting agents and fractionated radiation therapy: The role of the SDF-1/CXCR4 pathway. Int J Radiat Oncol Biol Phys 86: 777-784, 2013

22. Bottoni U, Bonaccorsi P, Devirgiliis V, Panasiti V, Borroni RG, Trasimeni G, Clerico R and Calvieri S: Complete remission of brain metastases in three patients with stage IV melanoma treated with BOLD and G-CSF. Jpn J Clin Oncol 35: 507-513, 2005.

23. Löwenberg B, van Putten W, Theobald M, Gmür J, Verdonck L, Sonneveld P, Fey M, Schouten H, de Greef G, Ferrant A, et al: Effect of priming with granulocyte colony-stimulating factor on the outcome of chemotherapy for acute myeloid leukemia. $\mathrm{N}$ Engl J Med 349: 743-752, 2003. 
24. Di Carlo E, Forni G, Lollini P, Colombo MP, Modesti A and Musiani P: The intriguing role of polymorphonuclear neutrophils in antitumor reactions. Blood 97: 339-345, 2001.

25. Koga Y, Matsuzaki A, Suminoe A, Hattori H and Hara T: Neutrophil-derived TNF-related apoptosis-inducing ligand (TRAIL): A novel mechanism of antitumor effect by neutrophils. Cancer Res 64: 1037-1043, 2004.

26. Gabrilovich DI and Nagaraj S: Myeloid-derived suppressor cells as regulators of the immune system. Nat Rev Immunol 9: $162-174,2009$.

27. Ohki Y, Heissig B, Sato Y, Akiyama H, Zhu Z, Hicklin DJ, Shimada K, Ogawa H, Daida H, Hattori K, et al: Granulocyte colony-stimulating factor promotes neovascularization by releasing vascular endothelial growth factor from neutrophils. FASEB J 19: 2005-2007, 2005.

28. Roskoski R Jr: Vascular endothelial growth factor (VEGF) signaling in tumor progression. Crit Rev Oncol Hematol 62: $179-213,2007$.

29. Heissig B, Hattori K, Dias S, Friedrich M, Ferris B, Hackett NR Crystal RG, Besmer P, Lyden D, Moore MA, et al: Recruitment of stem and progenitor cells from the bone marrow niche requires MMP-9 mediated release of kit-ligand. Cell 109: 625-637, 2002.
30. Hattori K, Heissig B, Tashiro K, Honjo T, Tateno M, Shieh JH, Hackett NR, Quitoriano MS, Crystal RG, Rafii S, et al: Plasma elevation of stromal cell-derived factor-1 induces mobilization of mature and immature hematopoietic progenitor and stem cells. Blood 97: 3354-3360, 2001.

31. Kioi M, Vogel H, Schultz G, Hoffman RM, Harsh GR and Brown JM: Inhibition of vasculogenesis, but not angiogenesis, prevents the recurrence of glioblastoma after irradiation in mice. J Clin Invest 120: 694-705, 2010

32. Prionas SD, Kowalski J, Fajardo LF, Kaplan I, Kwan HH and Allison AC: Effects of X irradiation on angiogenesis. Radiat Res 124: 43-49, 1990.

33. Heissig B,Hattori K,Friedrich M,Rafii S and WerbZ: Angiogenesis: Vascular remodeling of the extracellular matrix involves metalloproteinases. Curr Opin Hematol 10: 136-141, 2003.

34. Bergers G, Brekken R, McMahon G, Vu TH, Itoh T, Tamaki K, Tanzawa K, Thorpe P, Itohara S, Werb Z, et al: Matrix metalloproteinase-9 triggers the angiogenic switch during carcinogenesis. Nat Cell Biol 2: 737-744, 2000.

35. Ahn GO and Brown JM: Matrix metalloproteinase-9 is required for tumor vasculogenesis but not for angiogenesis: Role of bone marrow-derived myelomonocytic cells. Cancer Cell 13: 193-205, 2008. 